Widefield ScIENCE AND TeChNOLOGY For the SKA

SKADS CONFERENCE 2009

S.A. Torchinsky, A. van Ardenne, T. van den Brink-Havinga, A.J.J. van Es, A.J. Faulkner (eds.)

4-6 November 2009, Château de Limelette, Belgium

\title{
SKADS Array Configuration Study *
}

\author{
A. P. Lobanov ${ }^{1}$, D. V. Lal ${ }^{1 \star \star}$, and S. Jiménez-Monferrer ${ }^{1,2}$ \\ 1 Max-Planck-Institut für Radioastronomie, Auf dem Hügel 69, 53121 Bonn, Germany \\ 2 Universidad de Valencia, Dr. Moliner 50, 46100 Burjassot, Valencia, Spain
}

\begin{abstract}
Results from SKADS array configuration study are presented, focusing on a quantitative assessment of the effect of the array configuration on imaging performance of the SKA. The spatial dynamic range (SDR) and a measure of incompleteness of the Fourier domain coverage $(\Delta u / u)$ are employed as prime figures of merit (FoM). We conclude that $\Delta u / u \leq 0.03$ provides a benchmark at which the Fourier domain coverage will not be a dominant factor limiting imaging capabilities of the SKA. An algorithm for calculating the distribution of $\Delta u / u$ from interferometric data is described, and a set of FoM for evaluating array configurations is proposed.
\end{abstract}

\section{Introduction}

The Square Kilometre Array (SKA) will operate together with several new large optical, X-ray and Gamma-ray facilities. To make SKA both competitive and complementary to these large facilities, thorough design studies are needed, focused in particular on antenna configuration and its effect imaging performance of the array.

The SKA configuration must provide $u v$-coverages that satisfy several key requirements derived from the prime science goals of the instrument: (i) good snapshot and deep imaging over 1 degree field of view, (ii) low brightness temperature $\left(\mathrm{T}_{\mathrm{b}}\right)$ sensitivity for extended objects, (iii) dense core for transients/pulsar/SETI, and (iv) long baselines for milliarcsecond imaging. Combination of these requirements with the benchmark figures for the dynamic range of continuum $\left(10^{6}\right)$ and spectral line $\left(10^{5}\right)$ observations poses a substantial challenge for the array design and for the antenna distribution in particular.

Antenna configurations and resulting Fourier domain coverages ( $u v$-coverages) have been typically optimised by minimising sidelobes or providing a desired beam shape (cf., Cornwell 1986, Kogan 2000a, 2000b). These approaches rely implicitly on two basic assumptions.

1) The field of view is not crowded, i.e., the number of sources $N_{\mathrm{s}} \leq\left(B_{\max } / D_{\text {ant }}\right)^{2}$, where $D_{\text {ant }}$ is antenna diameter and $B_{\max }$ is the maximum baseline length.

2) Objects are marginally resolved, with majority of objects having sizes $\theta_{\mathrm{s}} \leq 2 \mathrm{HPBW} / \sqrt{\mathrm{SNR}}$, where HPBW marks the size of the synthesised beam and SNR is the design specification for signal-to-noise ratio of images. This warrants that the structural information can be recovered efficiently even if a substantial fraction of spatial frequencies is undersampled.

However, neither of these two assumptions will be correct for the SKA observations reaching down to microjansky levels

\footnotetext{
* This work was supported by the European Commission Framework Program 6, Project SKADS, Square Kilometre Array Design Studies (SKADS), contract no 011938.

$\star \star$ Present address: Harvard-Smithsonian Center for Astrophysics, MS-67, 60 Garden Street, Cambridge, MA 02138, USA
}

of flux density. In order to meet the design specifications for surveys and imaging, The SKA will need to have the capability of imaging adequately all spatial frequencies at which there is more than one sky object per primary beam area of the receiv ing element of the array. This complication requires additional quantitative constraints to be employed for assessing the quality of $u v$-coverage and its effect on structural sensitivity and $\Omega$ imaging capabilities of the array. The SKADS array configuration study has been aimed at identifying potential figures of merit suitable for this purpose and providing benchmark constraints for the SKA configuration design (Lal et al. 2009).

Alongside the dynamic range and shape of the synthesised point-spread-function (PSF or "synthesised beam"), one of the basic figures of merit $(\mathrm{FoM})$ characterising imaging performance of an interferometer is the spatial dynamic range (SDR). SDR provides an estimate of the range of spatial scales that can be reconstructed from interferometric data, which depends on a number of instrument parameters (Lobanov 2003), including the integration time of the correlator, channel bandwidth, and the coverage of the Fourier domain ( $u v$-coverage). The latter factor poses most stringent constraints on the configuration design, if the array imaging is required not be limited by the $u v$-coverage of observations.

It has been shown that the quality of the $u v$-coverage can be expressed by the $u v$-gap parameter or $\Delta u / u$, characterising the relative size of "holes" in the Fourier plane (Lobanov 2003). Basic analytical estimates indicate that the SKA should have $\Delta u / u \leq 0.2$ over the entire range of baselines to warrant that the $u v$-coverage will pose less stringent limits on the SDR compared to the limits arising from time and bandwidth smearing (Lobanov 2003). The SKADS array configuration study (Lal et al. 2009) has continued this effort by focusing on investigation of the effect of incompleteness of the $u v$-coverage on image properties.

We presents here the results from numerical simulations of interferometric data and analysis of the relation between the $u v$-gap parameter and image properties. Based on results of this analysis, we discuss potential application of the SDR and the 
$u v$-gap parameter for evaluation of site-specific array configurations proposed for the SKA.

\section{Structural sensitivity of an interferometer}

Structural sensitivity of an interferometer can be expressed by the spatial dynamic range (SDR) given by the ratio between the largest and smallest angular scales that can be adequately imaged. For filled apertures, SDR is essentially the ratio of field of view (FOV) to the characteristic size of the instrumental PSF. Incompleteness of $u v$-coverage results in reduced ability to recover structural information from intermediate angular scales which are formally within the range of scales sampled. This results in reduction of the SDR and introduction of scale dependent noise in images. Magnitude of these effects need to be assessed quantitatively and related to a parameter describing completeness of $u v$-coverage.

\section{1. uv-gap parameter}

The completeness of a $u v$-coverage can be expressed by the $u v$-gap parameter or $\Delta u / u$, characterising the relative size of "holes" in the Fourier plane. The " $u v$-gap" parameter can be defined as follows: $\Delta u / u=\left(u_{2}-u_{1}\right) / u_{1}$, where $u_{1}, u_{2}\left(u_{2}>u_{1}\right)$ are $u v$-radii of two adjacent $u v$-samples (sampling respective structural scales $\theta_{1,2}=1 / u_{1,2}$, with $\theta_{1}>\theta_{2}$ ). The $u v$-gap parameter can used to express structural sensitivity of an array. Ensuring a constant $\Delta u / u$ over the entire range of baselines, which will provide equal sensitivity to all spatial scales sampled by an interferometer and realize the full spatial dynamic range of the instrument.

\subsection{Spatial dynamic range}

The synthesised beam can be assumed to be approximated by a two-dimensional Gaussian (for instance, for a Gaussian bandpass and circular Gaussian tapering; $c f$., Bridle \& Schwab 1999). In this case, for structures partially resolved at $u_{2}$, the smallest resolvable size (or variation of the size) can be estimated by requiring that a difference in visibility amplitudes $V\left(u_{1}\right)$ and $V\left(u_{2}\right)$ can be detected at a given SNR. This approach is similar to the one applied to determining resolution limits of an interferometer (c.f., Lobanov et al. 2001), and for SNR $\gg 1$ it yields $\theta_{2} / \theta_{1} \approx(\pi / 4)[\ln 2 \ln (\mathrm{SNR})]^{-1 / 2}$. The ratio $\theta_{2} / \theta_{1}$ can be represented by the term $1+\Delta u / u$. With this term, the expression $\mathrm{SNR}_{\Delta u}=\exp \left[\pi^{2}(1+\Delta u / u)^{2} /(16 \ln 2)\right]$ gives the relation between $\Delta u / u$ and the SNR required for detecting changes in the visibility function for angular scales corresponding to the $\left(u_{1}, u_{2}\right)$ range. In case of a filled aperture, for which $\Delta u / u \rightarrow 0$, the corresponding $\mathrm{SNR}_{\Delta u}=\mathrm{SNR}_{\Delta u / u=0}=$ $\exp \left[\pi^{2} /(16 \ln 2)\right]$ and $\mathrm{SDR}_{\Delta u}=\mathrm{SDR}_{\mathrm{FOV}}$. For partially filled apertures, the ratio $\mathrm{SDR}_{\Delta u} / \mathrm{SDR}_{\mathrm{FOV}}$ can be estimated from the ratio $\mathrm{SNR}_{0} / \mathrm{SNR}_{\Delta u} /$, which then gives

$$
\mathrm{SDR}_{\Delta u}=\mathrm{SDR}_{\mathrm{FoV}} / \exp \left[\frac{\pi^{2}}{16 \ln 2} \frac{\Delta u}{u}\left(\frac{\Delta u}{u}+2\right)\right] \text {. }
$$

Strictly speaking, the $u v$-gap parameter is a function of the location $(u, \theta)$ in the $u v$-plane (with $\theta$ describing the position an-

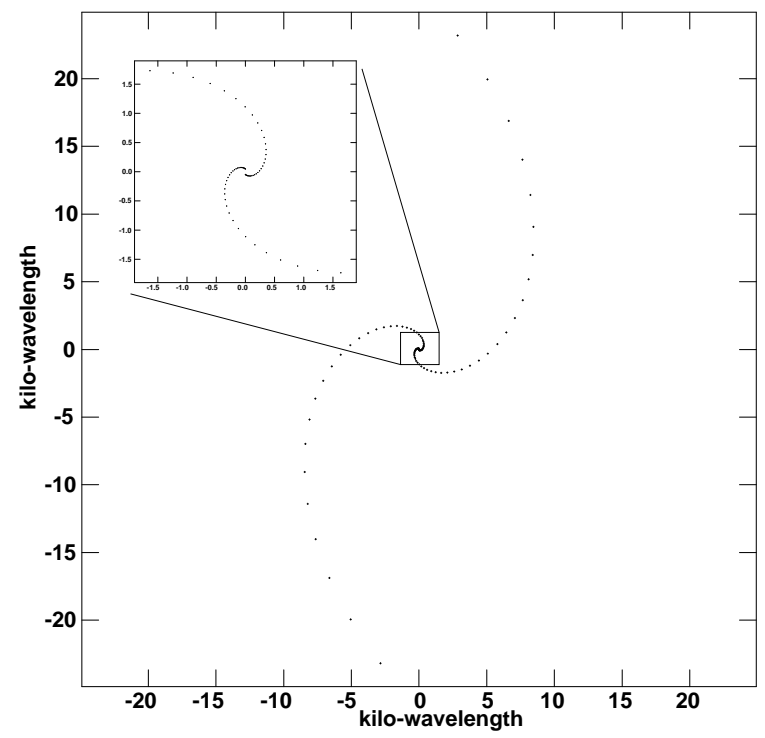

Fig. 1: An example of a simulated $u v$-coverage for a 2-minute long snapshot observation. The points outline relative locations of antenna stations. The largest baseline length, $B_{\max }=5 \mathrm{~km}$, and the number of antennas is $N=50$; In the simulations, only baselines to the central station have been considered, resulting in $\Delta u / u \equiv 0.19$ for a full track observation.

gle), and it should be represented by a density field in the $u v-$ plane.

For an idealised, circular $u v$-coverage obtained with a regular array (i.e. logarithmic-spiral) with $N$ stations organised in $M$ arms extending over a range of baselines $\left(B_{\min }, B_{\max }\right)$, the $u v$-gap can be approximated by $\Delta u / u \approx\left(B_{\max } / B_{\min }\right)^{\xi}-1$, with $\xi=M / N$ for baselines between antennas on a single arm, and $\xi=1 / N$ for all baselines.

The 1/ exp factor in the expression for $\mathrm{SDR}_{\Delta u}$, calculated for two $u v$-points $u_{1}$ and $u_{2}$, essentially provides an estimate of a fraction of power that can be recovered by the interferometer on angular scales $\left(\theta_{2}, \theta_{1}\right)$ from the sky brightness distribution. When an average value of $\Delta u / u$ over the entire $u v$-coverage is determined, the 1 / exp factor can be taken as a measure of ratio between the largest detectable structure and the primary beam (FOV) of individual array elements (under condition that the largest detectable size obtained from $\Delta u / u$ is smaller that the largest angular scale given by $\left.1 / u_{\min }\right)$.

For instruments with multi-frequency synthesis (MFS) implemented, $\Delta u / u$ should be substituted by $\Delta u / u-\Delta v_{\mathrm{mfs}}$, where, $\Delta v_{\mathrm{mfs}}$ is the fractional bandwidth over which the multifrequency synthesis is being performed. It should be noted that MFS will be not as effective improving $\Delta u / u$ for sources near the equator.

\section{Numerical simulations}

In order to provide a quantitative measure of the effect of array configuration on imaging performance of an interferometer, we have simulates a set of an idealised array configurations, each 
providing

$$
\frac{\Delta u}{u} \equiv \text { const }
$$

over the entire range of $u$ and $\theta$. A typical $u v$-coverage satisfying this condition is shown in Fig. 1.

We generate the test array configurations by considering an equiangular, planar logarithmic spiral and projecting this spiral on Earth's surface and determine the locations of individual stations (i.e., latitudes and longitudes) using World GEOD system 1984 (Heiskanen \& Moritz 1967). We apply Halley's third-order formula (Fukushima 2006), a modification of Borkowski's method (Borkowski 1989), to transform Cartesian to geodetic coordinates. This method is sufficiently precise; the maximum error of the latitude and the relative height is less than $6 \mu \mathrm{arcsec}$ for the range of height, $-10 \mathrm{~km}$ $\leq$ height $\leq 30,000 \mathrm{~km}$, and is stable in the sense that it converges for all coordinates including the near-geocentre region and near-polar axis region. We then transform these coordinates to obtain ECEF (Earth Centered Earth Fixed) coordinates using a glish script, and produce an input array configuration file. The actual dimensions of the simulated array are chosen such that baseline non-coplanarity is negligible and no $w$-term correction is required. This does not affect conclusions obtained from the simulations, which are generic and can be applied to evaluate imaging performance of any interferometric array of arbitrary configuration and extent.

We find that the best approach to generate array configurations providing $\Delta u / u \equiv$ const is to keep the baseline spread constant, and achieve different $\Delta u / u$ by changing the total number of antennas in the array. This method allows for maintaining a the same noise level for different array configurations. In order to ensure constant $\Delta u / u$ over the entire range of $u v$ distances, only baselines to the station at the origin of the array were considered. The generated visibility datasets probed the $u v$-gap parameter from 0.19 to 0.01 . The simulated integration time was set to 1 second, and the resulting sampling times ranged from 12.5 seconds (one $u v$-point every 12.5 seconds) for $N=50$ to 160 seconds for $N=640$. With these settings, visibility datasets were produced for full track $u v$-coverages for each of the array configurations considered. Further details of the numerical setup and simulation procedures are described in SKA Memo 107 (Lal et al. 2009).

The simulated data have been used for determining the PSF shape (see Fig. 2), r.m.s. noise, peaks surface brightness, and dynamic range of resulting images obtained from both direct Fourier inversion ("dirty" images, see Fig. 2) and by applying CLEAN deconvolution ("CLEAN" images). Values measured for these FoM are plotted in Fig. 3. The dependence of the r.m.s noise (and reciprocally, the dynamic range) on the $u v$-gap parameter changes at $\Delta u / u \approx 0.03$. At smaller values of $\Delta u / u$, the $u v$-coverage does not have a strong effect on the flux density recovered from the visibility data (this holds true for both dirty and CLEAN images). The requirement of $\Delta u / u \leq 0.03$ for the entire range of baselines can therefore be used as a benchmark requirement for designing the SKA configurations that would minimise the effect of $u v$-coverage on reconstructing the sky brightness distribution. It should be noted that this conclusion provides a strong benchmark, implying implementing $u v$-coverages with $u v$-gaps larger than 0.03 will limit the imaging capabilities of an array even for data processing with the present day convolution and imaging algorithms.

Further, more detailed investigations may be required in order to refine this conclusion and assess the full range of effects that may potentially affect the dynamic range and structural sensitivity derived from images obtained with different $u v$ coverages and different values of $\Delta u / u$. One possibility would be to use more elaborate and more realistic sky models coming from source simulations (e.g., O'Sullivan et al. 2009).

\section{Distribution of the uv-gap parameter}

The $\Delta u / u$ parameter can be applied for evaluating an arbitrary array configuration. Since almost all real $u v$-coverages deviate from circular symmetry, $\Delta u / u$ should be represented either by a two-dimensional distribution or by an average of that. The averaging can be made azimuthally (providing a profile of $\Delta u / u$ as a function of $u v$-distance) or both azimuthally and radially (giving a single value description of a given $u v$-coverage). For the averaged quantities, the respective dispersions can be used to quantify inhomogeneities in the $u v$-coverages.

For the purpose of a better graphical representation of the $u v$-gap distribution, a definition $\Delta u / u=\left(u_{2}-u_{1}\right) / u_{2}\left(u_{2} \geq\right.$ $\left.u_{1}\right)$ can be applied. This definition changes the range of $\Delta u / u$ from $[0, \infty]$ to $[0,1]$, with 0 corresponding to $\Delta u / u$ from two identical baselines. The maximum value $\Delta u / u=1$ is realized everywhere outside the area covered by the observation (for which $u_{2}=\infty$ can be assumed).

A feasible approach to determine the figures of merit based on $\Delta u / u$ is as follows:

1. Grid the $u v$-data into $N$ sectors, where the width of each sector is determined by the observing scan-length (the duration of a snapshot observation, or a typical length of a single scan in a synthesis observation).

2. For each individual sector (described by its central position angle, $\phi_{i}$ and width $2 \pi / N$ and containing $M u v$-points), determine $\Delta u / u\left(u, \phi_{i}\right)$ for all $M u v$-points. As was pointed above, $\Delta u / u\left(u, \phi_{i}\right)<1$ within the range $u_{1} \leq u \leq u_{M}$ and it is equal to unity elsewhere.

3. Plot the combined results for all sectors in polar or rectangular coordinates or perform averaging in azimuth $\left\langle\frac{\Delta u}{u}(u, \phi)\right\rangle_{\phi}$ and/or azimuth and radius $\left\langle\frac{\Delta u}{u}(u, \phi)\right\rangle_{u, \phi}$.

A detailed description of the calculation algorithm is given in Appendix 1 of SKA Memo 107 (Lal et al. 2009).

\subsection{Examples}

In order to represent a two-dimensional distribution $\Delta u / u$, the Voronoi tessellation can be applied to combine the $\Delta u / u$ calculated for the individual sectors. The Voronoi tessellation ("Voronoi diagram") uses partitioning of a plane with $n$ points into convex polygons such that each polygon contains exactly one generating point and every point in a given polygon is closer to its generating point than to any other (Okabe et al. 2000). The Voronoi diagram is sometimes also known as a Dirichlet tessellation. The cells are called Dirichlet regions, 

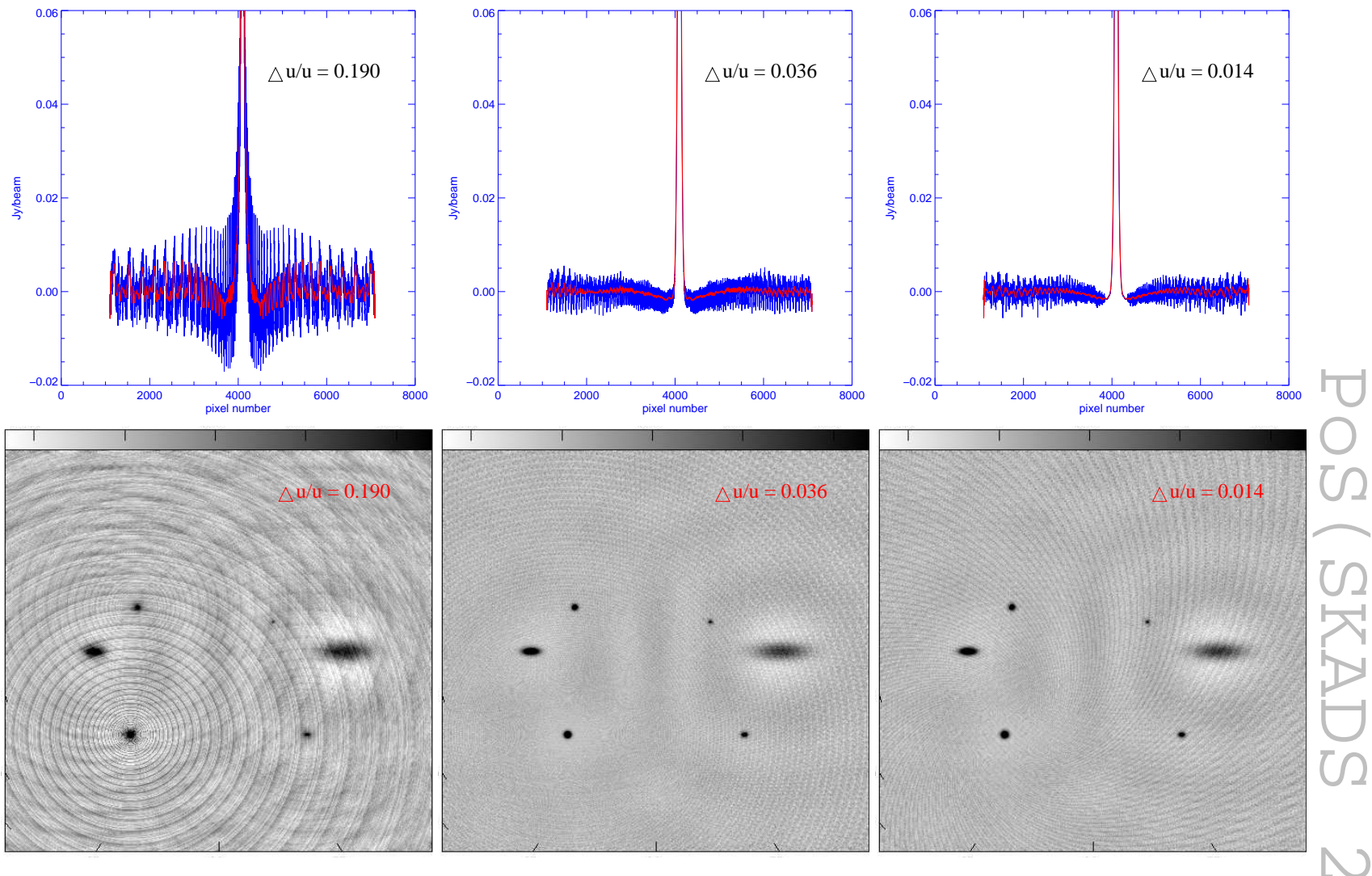

Fig. 2: Top row: One-dimensional profiles of restoring beams obtained from selected simulated datasets. The profiles are truncated at 0.06 of the beam peak in order to emphasise the difference in the sidelobes resulting from different $u v$-coverages. In each panel, the red curve represents the 30-pixel boxcar smoothing of the respective beam profile. Bottom row: Dirty images obtained from the simulated data. Each of the six panels is marked with the value of $\Delta u / u$ for the respective simulated dataset (Lal et al. 2009).

Thiessen polytopes, or Voronoi polygons. We use the algorithm by Okabe et al. (2000) to decompose the discrete set of $\frac{\Delta u}{u}\left(u v, \phi_{i}(i=1, N)\right.$ values into density plots that can be used as a graphical representation of the $u v$-gap distribution.

An example of a $u v$-coverage and the resulting twodimensional distribution and radial (azimuthally averaged) profile of $\Delta u / u$ is shown in Figs. 4-6. The overall (azimuthally and radially averaged) value of $\Delta u / u$ for this $u v$-coverage is $0.16 \pm 0.43$. The large dispersion of the average $\Delta u / u$ and the plots shown in Figs. 5-6 indicate that $\Delta u / u$ varies strongly with the baseline length and position angle. These variations should be minimised for the SKA configuration, taking into account the geographical location of the array, the different types of observations (e.g., snapshots, full track synthesis, multifrequency synthesis, etc.), and the range of declinations for which the imaging performance of the SKA should be optimised.

\section{Conclusions}

The SKADS array configuration study (Lal et al. 2009) has provided a tool for evaluating the effect of incomplete $u v$-coverage on imaging performance of the SKA, based on the $u v$-gap parameter $\Delta u / u$. A benchmark requirement of $\Delta u / u \leq 0.03$ de- rived can be used for initial assessment of array configuration proposed for the SKA, and should be further improved by simulations employing realistic sky brightness distributions and site-specific array configurations.

Based on the analysis undertaken as part of the SKADS array configuration study, an initial set of figures of merit (FoM) can be proposed for evaluating imaging performance of different array configurations for the SKA (see Table 1). The set of FoM proposed includes general performance metrics and does not include specific requirements on array configuration coming from individual SKA Key Science Projects. The critical issues for evaluating imaging performance provided by a given array configuration are:

1. Providing optimal shape of the point-spread-function (PSF).

2. Minimizing sidelobes of the PSF.

3. Evaluating sensitivity to all spatial scales sampled by the SKA.

Table 1 sumarizes a basic set of seven FoM suitable for evaluating the three conditions listed above. The FoM listed in 


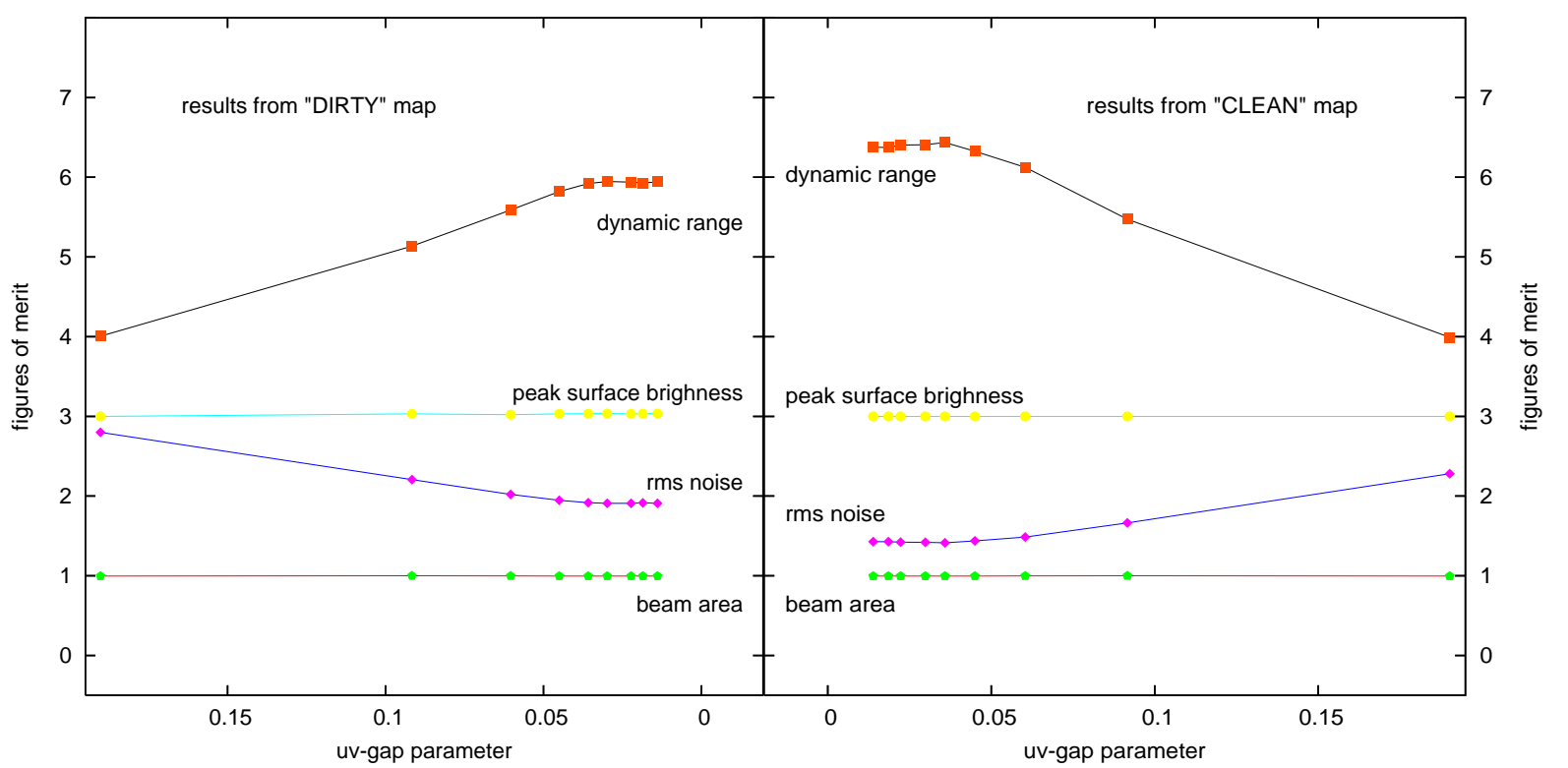

Fig. 3: Relative changes of different FoMs obtained from the dirty (left panel) and CLEAN (right panel) maps. The normalisation is done with respect to the FoM values determined from the dirty image with $\Delta u / u=0.01$, by normalising its beam area to unity, rms noise to the value of 2 , peak surface brightness to the value of 3 , and dynamic range to the value of 6 . The peak surface brightness recovered from different simulation runs remains nearly constant. The rms noise first decreases rapidly for smaller values of $\Delta u / u$, but the rate of this decrease is then remarkably reduced, implying that the $u v$-coverage does not affect strongly the image noise and dynamic range for array configurations with $\Delta u / u<0.03$. Note that the dynamic range is determined for a feature with the size substantially smaller than the largest angular scale for the $u v$-coverage with $\Delta u / u=0.19$, and thus the calculations are not affected by variable minimum $u v$-spacing in different simulation runs (Lal et al. 2009).

Table 1: Set of figures of merit for SKA configuration evaluation

\begin{tabular}{||l|l|ll|l||}
\hline \hline \multicolumn{1}{||c|}{ FoM } & \multicolumn{1}{|c|}{ Description } & \multicolumn{2}{c|}{ Name } & \multicolumn{1}{c|}{ Goal } \\
\hline \hline Point Spread & Major Axis & BMA, & $b_{\text {maj }}$ & \\
& Minor Axis & BMI, & $b_{\text {min }}$ & $b_{\text {min }} / b_{\text {maj }} \rightarrow 1$ \\
& P.A. of Major Axis & PAN, & $\theta_{\text {maj }}$ & \\
& PSF Shape & PSF, & $B(r, \theta)$ & $B(r, \theta) \rightarrow$ Gaussian \\
\hline PSF Sidelobes & Maximum Positive Sidelobe & MPS, & $\sigma_{+}$ & $\sigma_{+} \rightarrow 0$ \\
& Maximum Negative Sidelobe & MNS, & $\sigma_{-}$ & $\sigma_{-} \rightarrow 0$ \\
& Sidelobe RMS & RMS, & $\sigma_{\text {rms }}$ & $\sigma_{\text {rms }} \rightarrow 0$ \\
\hline UV Gap & Integrated Value & UVG, & $\Delta u / u$ & $\langle\Delta u / u\rangle_{u, \theta} \rightarrow 0$ \\
& Dispersion & UVD, & $\Delta u / u(u, \theta)$ & $\sigma_{\Delta u / u} \rightarrow 0$ \\
\hline \hline
\end{tabular}

Table 1 are described in polar coordinates in the $u v$-plane $(u, \theta)$ and image plane $(r, \theta)$.

\section{References}

Borkowski, T.J. 1989, Bull. Géod. 63, 50

Bridle, A. H., Schwab, F. R., 1999, ASP Conf. Series, v. 180, p. 371

Cornwell, T.J. (1986) “Crystalline antenna arrays,” MM Array Memo 38

Fukushima, T. 2006, Journal of Geodesy, 79, 689

Heiskanen W.A., Moritz, H. 1967, "Physical geodesy," WH Freeman, New York

Kogan, L. 2000a, ASP Conf. Series, vol. 217, p. 348
Kogan, L. 2000b, IEEE transactions on antennas and propagation, vol. 48, p. 1075

Lal, D. V., Lobanov, A. P., Jímenez-Monferrer, S. 2009, SKA Memo 107 www. skatelescope.org

Lobanov, A. P., Gurvits, L. I., Frey, S., Schilizzi, R. T., Kawaguchi, N. \& Pauliny-Toth, I. I. K. 2001, ApJ, 547, 714

Lobanov, A. P., 2003, SKA Memo 38 www.skatelescope. org

Okabe, A., Boots, B., Sugihara, K. \& Chiu, S.N. (2000) "Spatial tessellations - Concepts and applications of Voronoi diagrams," John Wiley (2nd edition)

O’Sullivan, S.P., Stil, J.M., Taylor, A.R., Ricci, R., Grant, J.K. \& Shorten, K. 2009, SKADS Memo 31 www . skads-eu . org 


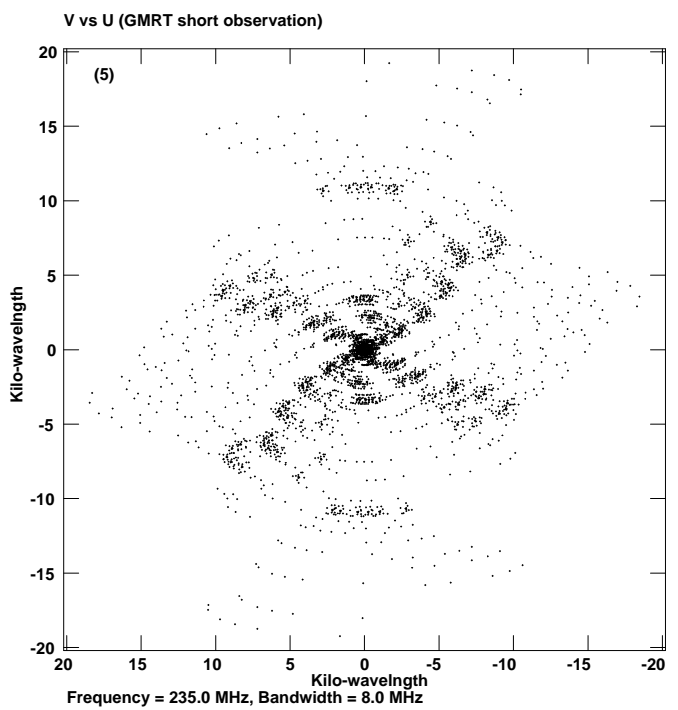

Fig. 4: An example $u v$-coverage from a short observation with the Giant Meter Wavelength Telescope (GMRT) used for calculation of $\Delta u / u$.
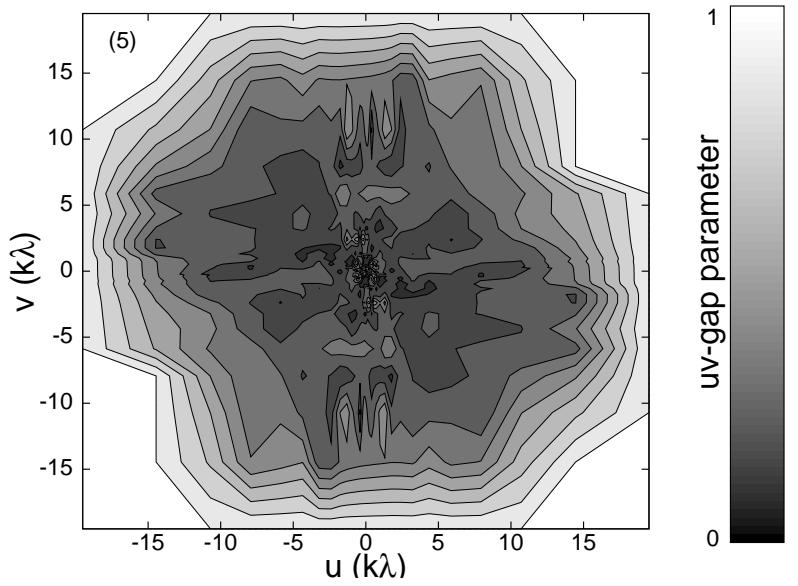

Fig. 5: Density plot of $\frac{\Delta u}{u}(u, \phi)$ distribution calculated using Voronoi tessellation from the $u v$-coverage shown in Fig 4.

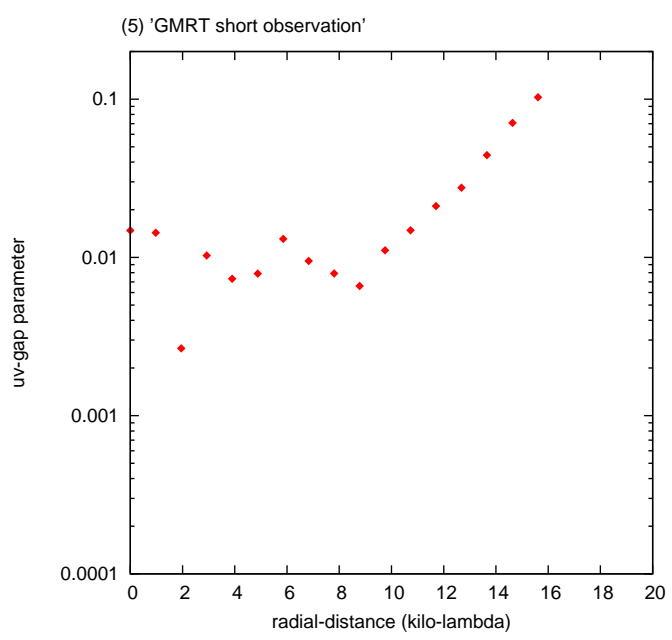

Fig. 6: Azimuthally averaged profile of $\Delta u / u$ from the density plot of shown in Fig. 5. The mean $\Delta u / u$ for this profile is $0.16 \pm 0.43$, with the large dispersion implying uneven sensitivity at different scales )(note a strong gradient of $\Delta u / u$ with increasing radial $u v$-distance). 